\title{
Some Charge Control Criteria for Focused Ion Beam Milling of Insulators and Biological Specimens using a Quanta 3D DualBeam ESEM
}

\author{
D.J. Stokes, ${ }^{*}$ F. Morrissey, ${ }^{* *}$ W. R. Knowles ${ }^{* * *} \&$ A.M. Donald* \\ * Biological \& Soft systems, Cavendish Laboratory, University of Cambridge, Dept. of Physics, \\ Madingley Road, Cambridge, CB3 0HE, UK \\ ** FEI Company, The Aztec Centre, Bristol, BS32 4TD, UK \\ ${ }^{* * *}$ FEI Company, One Corporation Way, Centennial Park, Peabody, MA, USA
}

There is currently great interest in applying FIB technology in diverse fields outside the traditional semi-conductor arena, including advanced study of biological microstructure. However, milling of such insulating materials with a positive ion beam in high vacuum requires some form of charge control. For single beam FIB and FEG SEM FIB, a specialised electron source is needed/preferable. However, for a tungsten FIB (E)SEM, such as the Quanta 3D, the primary electron beam can supply low energy, defocused electrons in sufficient measure to confer stability and enable accurate milling and vapor deposition, even when using a large ion beam current.

In selecting an appropriate electron beam current $I_{b(e)}$, it is useful to have a rule-of-thumb for the positive current $I_{b(i o n)}$ to be counteracted, taking into account both the incoming ions and positive charge due to secondary electron emission at the surface. Empirically, this is taken to be:

$$
I_{b(e)} \sim 3 I_{b(\text { ion })} \quad \text { Equation } 1
$$

This criterion then needs to be related to the areas over which the beams are being applied: in the other words the current densities. Ion beam current density $J_{b(i o n)}$ can easily be calculated from the $x$ $y$ dimensions of the milling pattern. Hence:

$$
J_{b(\text { ion })}=I_{b(\text { ion })} / a \quad(\text { with area } a=x \times y) \quad \text { Equation } 2
$$

We must then correlate these dimensions with the area covered by the defocused electron beam. The required radius $r$ of the electron beam for a given area $a$ is given by:

$$
r=\sqrt{ }\left(a^{2} / \pi\right)
$$

Equation 3

Finally, we must deduce the defocus $d$ required to produce a given spot size. Defocus is defined as the vertical distance through which the focal length of the beam is changed, analogous to moving the specimen a given distance without refocusing (see Figure 1). Using a little geometry, the required defocus $d$ can be found from the following expression, where $d_{w}$ is the working distance:

$$
\frac{d_{w}}{(100 / r)-1}
$$

Equation 4

These equations enable us to calculate the electron current density required to match the positive current resulting from ion irradiation, provided that the aspect ratio of the milling pattern is sensible. For very high aspect ratios, alternative methods may have to be used. 
Figure 2(a) shows a highly insulating (polymeric) specimen FIB milled with no charge control applied, while (b) shows the vast improvement when charge neutralization is used according to the above criteria. In this way, a number of challenging biological specimens have been successfully milled [1] enabling us to gain new insights by, for example, 3D reconstruction from 2D data sets.

\section{References}

[1] D.J. Stokes, J.R. Tong, J. Juhasz, P.A. Midgley \& S.M. Best, elsewhere in these proceedings [2] This work was funded by FEI Company. Helpful discussions with J. Rickard and other colleagues at the Cavendish Lab are gratefully acknowledged.
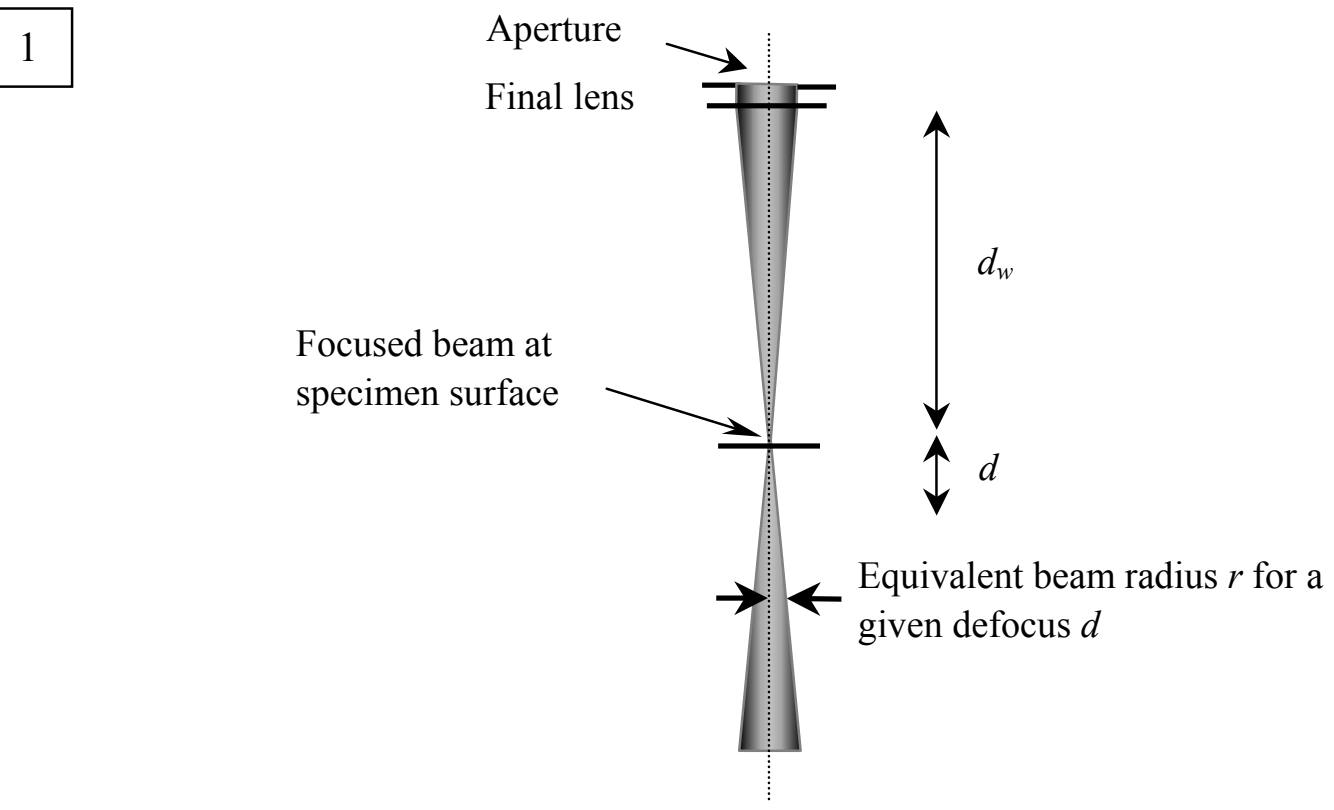

Figure 1. Schematic diagram showing some of the parameters needed for Equation 4.
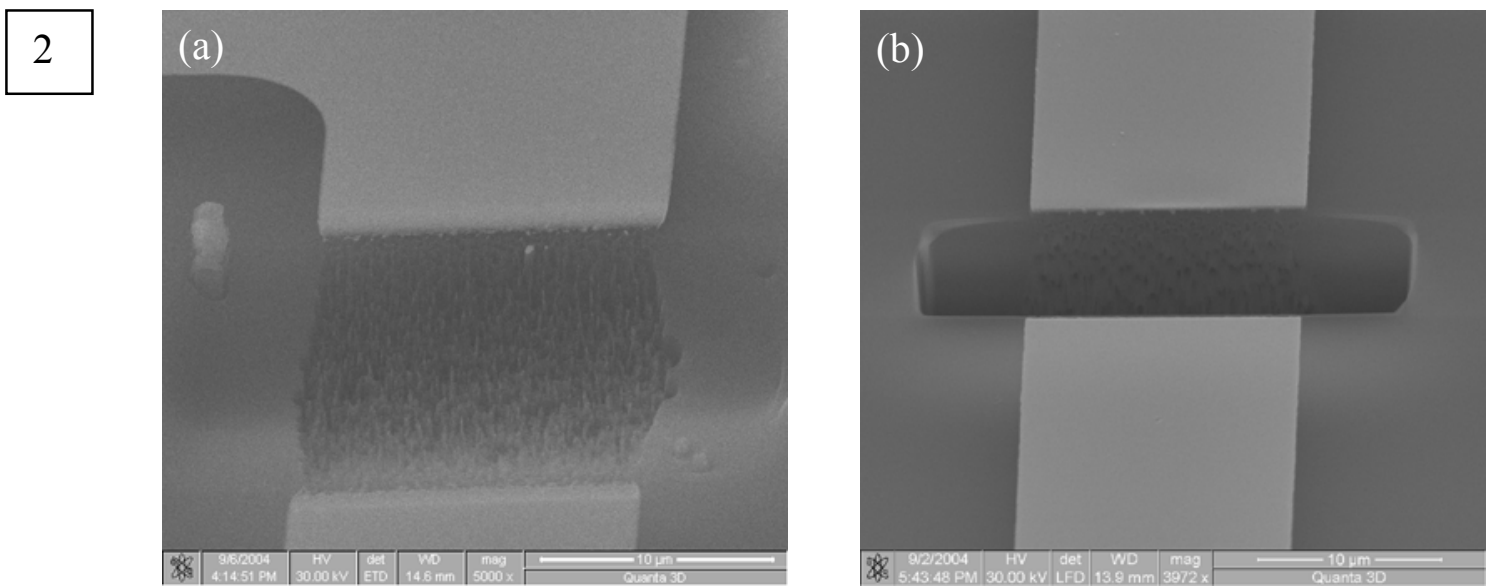

Figure 2. (a) Charge-induced ion beam drift results in a distorted FIB cut. (b) Application of charge neutralization, as described above, ensures that a clean cut is made. 